\title{
Solvable K-essence Cosmologies and Modified Chaplygin Gas Unified Models of Dark Energy and Dark Matter
}

\author{
M. Sharif ${ }^{1}{ }^{*}$ K.R. Yesmakhanova ${ }^{2}$, S. Rani ${ }^{1} \nmid$ R. Myrzakulov ${ }^{2} \ddagger$ \\ ${ }^{1}$ Department of Mathematics, University of the Punjab, \\ Quaid-e-Azam Campus, Lahore-54590, Pakistan. \\ ${ }^{2}$ Eurasian International Center for Theoretical Physics, \\ Eurasian National University, Astana 010008, Kazakhstan
}

\begin{abstract}
This paper is devoted to the investigation of modified Chaplygin gas model in the context of solvable k-essence cosmologies. For this purpose, we construct equations of state parameter of this model for some particular values of the parameter $n$. The graphical behavior of these equations are also discussed by using power law form of potential. The relationship between k-essence and modified Chaplygin gas model shows viable results in the dark energy scenario. We conclude that the universe behaves as a cosmological constant, quintessence phase or phantom phase depending upon $n$.
\end{abstract}

\section{Introduction}

The accelerating cosmic expansion of the universe, motivated by the mysterious dark energy (DE) idea, was noticed in 1998 through supernovae experiments [1]. A number of various cosmological observations claim that this energy fills the space but yet there is no clue about its identity. The expansion of the universe started to accelerate when the energy density of matter was overcome by the DE. The evolution of DE density is governed by an equation of state parameter, $\omega=p / \rho$. This parameter has raised recently many interesting features for accelerating expansion of the universe. Some of them are as follows:

- Qunitessence region obeys $-1<\omega \leq-1 / 3$ [2],

\footnotetext{
*msharif.math@pu.edu.pk

${ }^{\dagger}$ shamailatoor.math@yahoo.com

${ }^{\ddagger}$ rmyrzakulov@csufresno.edu
} 
- Cosmological constant is considered to be the simplest case for DE for which $\omega=-1$,

- For phantom phase, the EoS parameter follows the domain $\omega<-1$ [3].

- Qunitum model inherits both of the properties of quintessence and phantom phases by crossing the phantom divide line $\omega=-1$ [4].

Modified Chaplygin Gas (MCG) model [5] is one of the proposed models to discuss the DE dominated phase for accelerating expansion of the universe. It is a single fluid model to unify the DE and dark matter. This model behaves as dark matter when its energy density evolves as $\rho \propto a^{-3}$ in the early times of the universe (where the scale factor $a$ is a function of time), whereas the constant energy density behaves as DE era in late time acceleration. Thus the MCG model provides unification between dark matter and DE [6]. The scalar field model is another category to discuss the DE scenario. Recently, k-essence [7] gains an important role to explore the dynamics of the universe. It inherits a kinetic term which deals with late time inflation and early time acceleration named as k-inflation [8].

Another DE model, g-essence, is a general essence model reduced in two models: k-essence (kinetic quintessence) and f-essence (fermionic k-essence) [9]. Razina et al. [10] explored the relationship between fermions and scalar fields by taking two gessence models. They concluded that g-essence describes the accelerating expansion of the universe. Tsyba et al. [11] reconstructed the interaction among Chaplygin gas, Generalized Chaplygin gas as well as fermions and found EoS parameter for particular f-essence models. Myrzakulov [12] discussed the fermionic k-essence to explore accelerating expansion of the universe. Jamil et al. [13] investigated the behavior of solvable f-essence cosmology in MCG scenario.

In this paper, we study the purely kinetic k-essence cosmology by using the phenomenon of MCG model. We obtain a non-linear differential equation which depends upon the parameter $n$. The EoS parameter is evaluated by using some particular values of $n$ and is displayed graphically to investigate the dynamics of the universe. The power law form of potential function is used to obtain the kinetic term of purely kinetic k-essence.

The paper is organized as follows. In the next section, we present some basics of k-essence model and the MCG model. In section 3, the EoS parameter is constructed for different cases and their graphical representation is discussed. Section 4 describes the results for some other forms of solvable k-essence cosmology. In the last section, we summarize and conclude the results.

\section{K-essence and MCG Formalism}

Here, we consider the class of models described by the following action

$$
S=\int d^{4} x \sqrt{-g}[R+2 K(X, \phi)]
$$

where $\phi$ is the scalar field, $R$ is the Ricci scalar,

$$
X=-0.5 g^{\mu \nu} \nabla_{\mu} \phi \nabla_{\nu} \phi
$$


is the canonical kinetic energy and $g_{\mu \nu}$ is the metric tensor, $\nabla_{\mu}$ is the covariant derivative associated with metric $g_{\mu \nu}$. We are interested in flat, homogeneous and isotropic FRW universe described by the line element

$$
d s^{2}=-d t^{2}+a^{2}\left(d x^{2}+d y^{2}+d z^{2}\right),
$$

where $a(t)$ is the scale factor. The k-essence equations corresponding to the action (2.1) read as

$$
\begin{aligned}
3 H^{2}-\rho & =0, \\
2 \dot{H}+3 H^{2}+p & =0, \\
K_{X} \ddot{\phi}+\left(\dot{K}_{X}+3 H K_{X}\right) \dot{\phi}-K_{\phi} & =0, \\
\dot{\rho}+3 H(\rho+p) & =0 .
\end{aligned}
$$

Here dot denotes derivative with respect to cosmic time, the Hubble rate is $H=\dot{a} / a$, the kinetic term, the energy density and the pressure, respectively, are

$$
X=0.5 \dot{\phi}^{2}, \quad \rho=2 X K_{X}-K, \quad p=K .
$$

It was shown in [14] that for this model the speed of propagation of scalar perturbations (speed of sound), $c_{s}$, is given by

$$
c_{s}^{2}=\frac{p_{X}}{\rho_{X}}=\frac{p_{X}}{p_{X}+2 X p_{X}} .
$$

In the case of purely kinetic k-essence, $K=K(X)$, Eq.(2.5) takes the form

$$
K_{X} \ddot{\phi}+\left(\dot{K}_{X}+3 H K_{X}\right) \dot{\phi}=0,
$$

yielding the general solution

$$
X K_{X}^{2}=k a^{-6}
$$

with $k \geq 0$ [15, 16].

The EoS of the MCG DE model is [17]

$$
p=A \rho-\frac{B}{\rho^{\alpha}},
$$

where $A$ and $B$ are positive constants and $0 \leq \alpha \leq 1$. Using Eqs.(2.6) and (2.10), the MCG energy density and pressure evolve as

$$
\begin{aligned}
& \rho=\left[B(1+A)^{-1}+C a^{-3(1+\alpha)(1+A)}\right]^{\frac{1}{1+\alpha}} \\
& p=\left[A C a^{-3(1+\alpha)(1+A)}-B(1+A)^{-1}\right]\left[B(1+A)^{-1}+C a^{-3(1+\alpha)(1+A)}\right]^{-\frac{\alpha}{1+\alpha}}
\end{aligned}
$$

In this case, the EoS parameter is

$$
\omega=\frac{A C a^{-3(1+\alpha)(1+A)}-B(1+A)^{-1}}{B(1+A)^{-1}+C a^{-3(1+\alpha)(1+A)}} .
$$

Here we assume the potential in the form of power law [18]

$$
\phi=\phi_{0} t^{\beta} .
$$

Using the above equation, the kinetic term takes the form

$$
X=0.5 \phi_{0}^{2} \beta^{2} t^{2(\beta-1)} .
$$

For the sake of simplicity, we assume $\phi_{0}=1$. 


\section{Solvable k-essence cosmology: $K=F(X)$}

In this section, we would like to consider the case [12] when

$$
K=F(X)
$$

that is the purely kinetic k-essence case. We solve the following system

$$
\begin{aligned}
\rho & =2 X F_{X}-F \\
F & =A \rho-\frac{B}{\rho^{\alpha}} .
\end{aligned}
$$

Solving Eq.(3.2) for $F$, it follows that

$$
F=E \sqrt{X}+\frac{\sqrt{X}}{2} \int \frac{\rho}{X^{1.5}} d X
$$

where $E$ is an integration constant. Equations (3.3) and (3.4) give

$$
E \sqrt{X}+\frac{\sqrt{X}}{2} \int \frac{\rho}{X^{1.5}} d X=A \rho-\frac{B}{\rho^{\alpha}},
$$

which has the solution

$$
(1+A) \rho^{1+\alpha}-(W X)^{\frac{n(1+\alpha)}{2 \alpha}} \rho^{n(1+\alpha)}-B=0,
$$

where $W$ is a constant and

$$
n=\frac{\alpha(1+A)}{A+\alpha(1+A)} .
$$

From Eq.(3.6), it follows that $A$ and $\alpha$ are related by

$$
A=-\frac{(n-1) \alpha}{(n-1) \alpha+n} \quad \text { or } \quad \alpha=-\frac{n A}{(n-1)(1+A)} .
$$

The search for the analytical solutions of Eq.(3.5) is a hard job. We would like to find particular solutions for some values of $n$ to get insights of the model. It is mentioned here that the values of constants [13, 19] in the models are taken in such a way that we obtain an expanding universe with acceleration.

\subsubsection{Example 1: $n=0$}

First, we consider the particular value $n=0$. It follows from Eq.(3.6) that this value yields two cases, either $\alpha=0$ or $A=-1$.

\section{Case 1}

Let us consider the case $\alpha=0$. Consequently, Eqs.(3.2) and (3.3) take the form

$$
\rho=2 X F_{X}-F, \quad F=A \rho-B .
$$




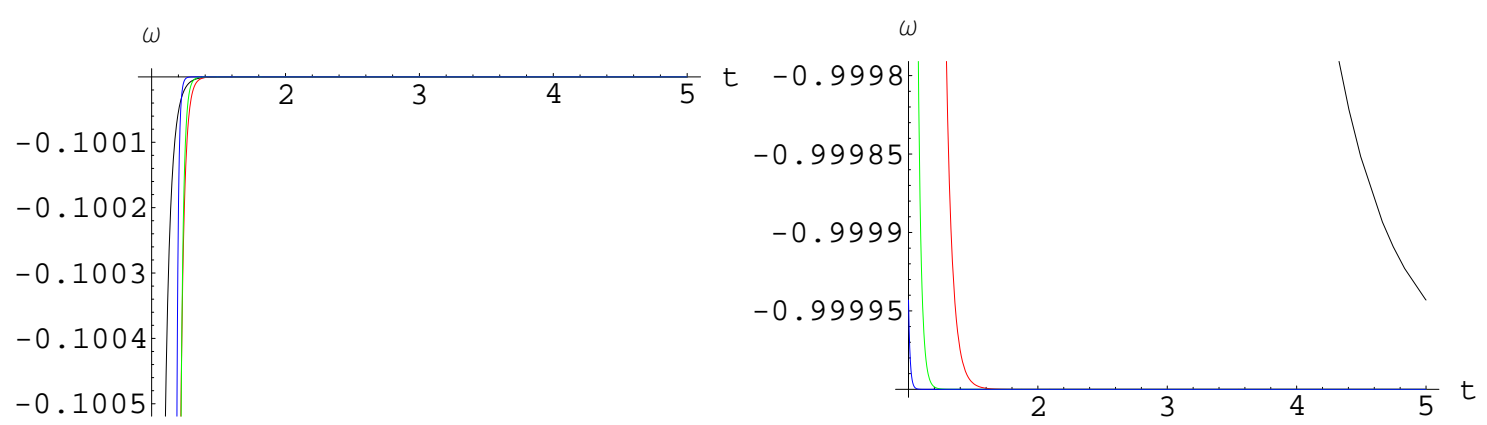

Figure 1: Plot of $\omega$ versus $t$ with $A=-0.1, W=0.2, B=0.5$. In the left graph, $\beta=-2$ (black), $\beta=-4$ (red), $\beta=-6$ (green), $\beta=-10$ (blue) and in right graph $\beta=2$ (black), $\beta=4$ (red), $\beta=6$ (green), $\beta=10$ (blue).

Solving the above equations, the equation for $\rho$ reads as

$$
(1+A) \rho-(W X)^{\frac{1+A}{2 A}}-B=0,
$$

which yields

$$
\rho=(1+A)^{-1}\left[B+(W X)^{\frac{1+A}{2 A}}\right] .
$$

The expression for pressure becomes

$$
p=(1+A)^{-1}\left[-B+A(W X)^{\frac{1+A}{2 A}}\right] .
$$

The corresponding EoS parameter is given by

$$
\omega=A-\frac{B(1+A)}{B+(W X)^{\frac{1+A}{2 A}}} .
$$

Using Eq.(2.15), the above equation can be written as

$$
\omega=A-\frac{B(1+A)}{B+\left(W \beta^{2} t^{2(\beta-1)} / 2\right)^{\frac{1+A}{2 A}}} .
$$

The behavior of EoS parameter $\omega$ in terms of time is shown in Figure 1 which indicates the cosmological evolution. For $\beta>0$, initially $\omega$ shows the DE phase of the universe dominated by quintessence. As the value of $\beta$ increases, the EoS parameter becomes constant at $\omega=-1$ indicating the universe behavior like cosmological constant. On the other hand, for $\beta<0$, the EoS parameter possesses negative values and becomes constant at -0.1 . It presents a universe which just evolves from matter dominated universe $(\omega=0)$ to $\mathrm{DE}$ energy dominating universe.

\section{Case 2}

Now we consider the case when $A=-1$. The corresponding equations (3.2) and (3.3) read as

$$
\rho=2 X F_{X}-F, \quad F=-\rho-B \rho^{-\alpha},
$$




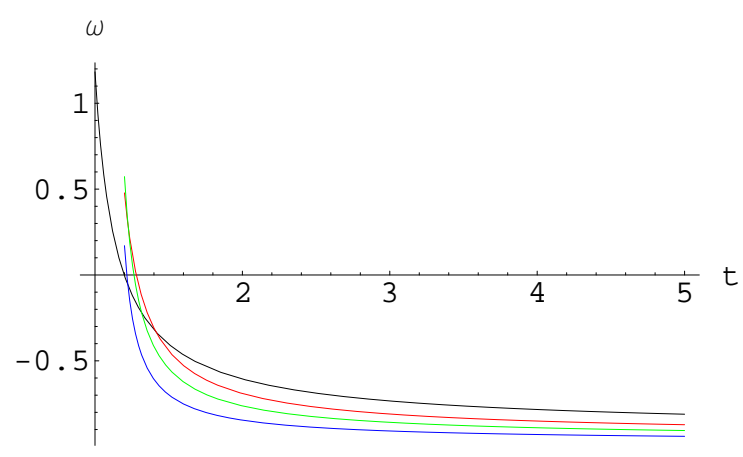

Figure 2: Plot of $\omega$ versus $t$ with $W=0.2$ and $\beta<0$.

which lead to

$$
\alpha B \ln \rho-(1+\alpha)^{-1} \rho^{1+\alpha}+\ln (W X)^{\frac{B}{2}}=0 .
$$

Consider some particular solutions of this equation.

i) If $\alpha=0$, then we have

$$
\rho=\ln (W X)^{\frac{B}{2}}
$$

and for the pressure

$$
p=\ln (W X)^{-\frac{B}{2}}-B .
$$

The corresponding EoS parameter in terms of potential is given by

$$
\omega=-1-\frac{2}{\ln \left[W \beta^{2} t^{2(\beta-1)} / 2\right]} .
$$

The EoS parameter $\omega$ shows the radiation and matter dominated universe for $t<1.3$ as shown in Figure 2. After this interval, the universe converges to DE dominated phase lying in the quintessence region as $-1<\omega \leq-1 / 3$.

ii) For $\alpha=-1$, the energy density and pressure become

$$
\rho=(W X)^{\frac{B}{2(1+B)}}, \quad p=-(1+B)(W X)^{\frac{B}{2(1+B)}} .
$$

The EoS parameter takes the following form

$$
\omega=-1-B .
$$

For this case, the behavior of the universe depends upon the value of constant $B$. If we take $B=0$, the universe converges to DE phase dominated by cosmological constant. For $B>0$, the universe meets the phantom region while $B<0$ shows a quintessence DE universe.

\subsubsection{Example 2: $n=1$}

If $n=1$, Eq.(3.6) yields $A=0$. This case corresponds to the generalized Chaplygin gas model [16] for which the expressions of $\rho$ and $p$ are as follows

$$
\begin{gathered}
\rho=B^{\frac{1}{1+\alpha}}\left[1-(W X)^{\frac{1+\alpha}{2 \alpha}}\right]^{\frac{-1}{1+\alpha}}, \\
p=-B^{\frac{1}{1+\alpha}}\left[1-(W X)^{\frac{1+\alpha}{2 \alpha}}\right]^{\frac{\alpha}{1+\alpha}} .
\end{gathered}
$$




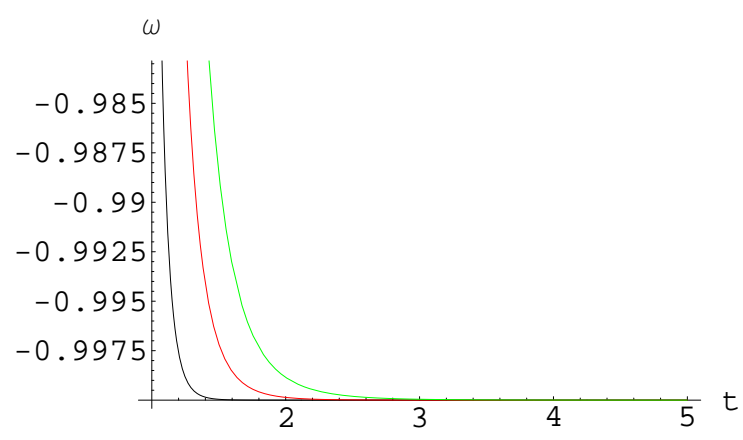

Figure 3: Plot of $\omega$ versus $t$ with $W=0.2, \beta=-2$ and $\alpha=0.2$ (black), $\alpha=0.4$ (red), $\alpha=0.6$ (green).

The corresponding EoS parameter is given by

$$
\omega=-1+(W X)^{\frac{1+\alpha}{2 \alpha}} .
$$

Inserting the value of $X$, Eq.(3.22) becomes

$$
\omega=-1+\left(\frac{W \beta^{2} t^{2(\beta-1)}}{2}\right)^{\frac{1+\alpha}{2 \alpha}} .
$$

Figure 3 shows the graphical behavior of the EoS parameter with respect to evolving time. The universe acquires quintessence region initially. As the time elapses, it becomes constant at $\omega=-1$ showing a universe dominated by the cosmological constant.

\subsubsection{Example 3: $n=2$}

Now we consider the case when $n=2$. Here $A$ and $\alpha$ are related by the formula

$$
A=-\frac{\alpha}{\alpha+2} \quad \text { or } \quad \alpha=-\frac{2 A}{1+A} .
$$

The equation for $\rho(3.5)$ takes the form

$$
(1+A) \rho^{1+\alpha}-(W X)^{\frac{1+\alpha}{\alpha}} \rho^{2(1+\alpha)}-B=0,
$$

which has the solution

$$
\rho=(W X)^{-\frac{1}{\alpha}}\left[\frac{1+A}{2} \mp \frac{1}{2} \sqrt{(1+A)^{2}-4 B(W X)^{\frac{1+\alpha}{\alpha}}}\right]^{\frac{1}{1+\alpha}}
$$

or

$$
\rho=(W X)^{-\frac{1}{\alpha}}\left\{\frac{1}{2+\alpha}\left[1 \mp \sqrt{1-B(2+\alpha)^{2}(W X)^{\frac{1+\alpha}{\alpha}}}\right]\right\}^{\frac{1}{1+\alpha}}
$$

The pressure is given by

$$
p=-\frac{\alpha(W X)^{-\frac{1}{\alpha}}}{\alpha+2}\left\{\frac{1}{2+\alpha}\left[1 \mp \sqrt{1-B(2+\alpha)^{2}(W X)^{\frac{1+\alpha}{\alpha}}}\right]\right\}^{\frac{1}{1+\alpha}}
$$




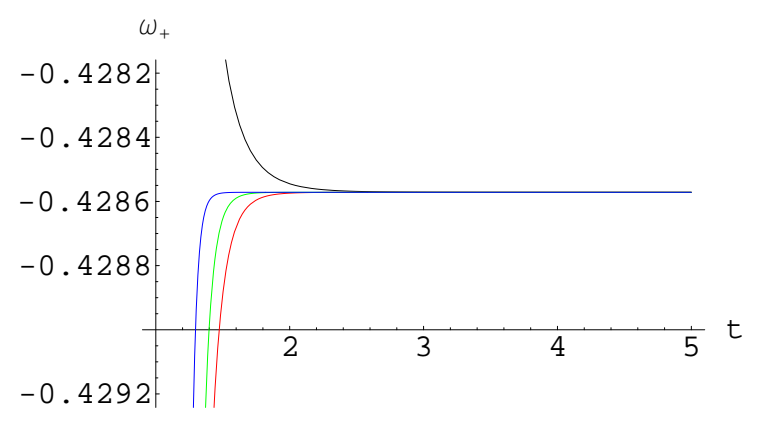

Figure 4: Plot of $\omega$ versus $t$ with $W=0.2, B=-0.1, \alpha=1.5$ and $\beta<0$.

$$
-B W X\left\{\frac{1}{2+\alpha}\left[1 \mp \sqrt{1-B(2+\alpha)^{2}(W X)^{\frac{1+\alpha}{\alpha}}}\right]\right\}^{-\frac{\alpha}{1+\alpha}} .
$$

The corresponding EoS parameter is

$$
\omega=-\frac{\alpha}{\alpha+2}-B(W X)^{\frac{1+\alpha}{\alpha}}\left\{\frac{1}{1+\alpha}\left[1 \mp \sqrt{1-B(2+\alpha)^{2}(W X)^{\frac{1+\alpha}{\alpha}}}\right]\right\}^{-1} .
$$

Inserting the value of potential and after simplifying the positive root, it yields

$$
\omega_{+}=-\frac{\alpha}{\alpha+2}-\frac{2 B(1+\alpha)\left(W \beta^{2} t^{2(\beta-1)}\right)^{\frac{1+\alpha}{\alpha}}}{2^{\frac{1+3 \alpha}{\alpha}}-B(\alpha+2)^{2}\left(W \beta^{2} t^{2(\beta-1)}\right)^{\frac{1+\alpha}{\alpha}}} .
$$

For this case, the EoS parameter results a universe having dynamics of quintessence of DE scenario shown in Figure 4. It becomes constant at $\omega_{+}=-0.4285$ with the passage of time. The negative root of Eq.(3.29) does not give any fruitful result.

\subsubsection{Example 4: $n=0.5$}

If $n=0.5$ then $A$ and $\alpha$ satisfy the relation

$$
A=-\frac{\alpha}{\alpha-1} \quad \text { or } \quad \alpha=\frac{A}{1+A}
$$

The equation (3.5) becomes

$$
(1+A) \rho^{1+\alpha}-(W X)^{\frac{1+\alpha}{4 \alpha}} \rho^{0.5(1+\alpha)}-B=0 .
$$

The solution of this equation reads as

$$
\rho=[2(1+A)]^{-\frac{2}{1+\alpha}}\left[(W X)^{\frac{1+\alpha}{4 \alpha}} \pm \sqrt{(W X)^{\frac{1+\alpha}{2 \alpha}}+4 B(1+A)}\right]^{\frac{2}{1+\alpha}}
$$

or

$$
\rho=\left(\frac{2}{1-\alpha}\right)^{-\frac{2}{1+\alpha}}\left[(W X)^{\frac{1+\alpha}{4 \alpha}} \pm \sqrt{(W X)^{\frac{1+\alpha}{2 \alpha}}+\frac{4 B}{1-\alpha}}\right]^{\frac{2}{1+\alpha}}
$$




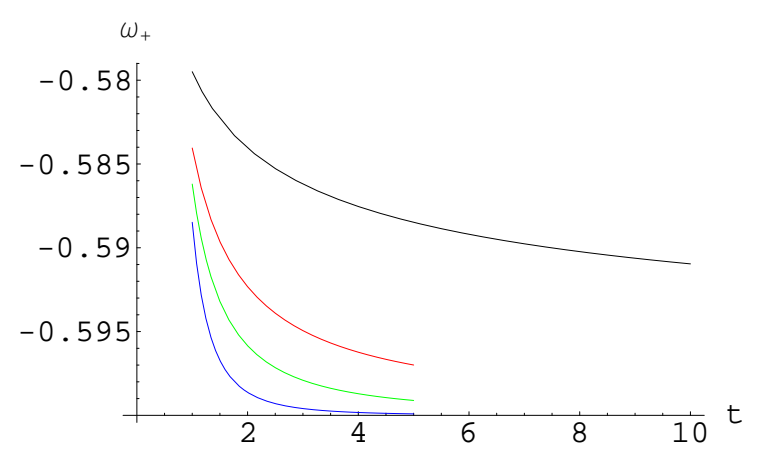

Figure 5: Plot of polytropic k-essence $\omega_{+}$versus $t$ with $W=0.2, B=-0.1, \alpha=$ -1.5 and $\beta>0$.

The pressure is given by

$$
\begin{aligned}
& p=\frac{\alpha}{1-\alpha}\left(\frac{2}{1-\alpha}\right)^{\frac{-2}{1+\alpha}}\left[(W X)^{\frac{1+\alpha}{4 \alpha}} \pm \sqrt{(W X)^{\frac{1+\alpha}{2 \alpha}}+\frac{4 B}{1-\alpha}}\right]^{\frac{2}{1+\alpha}} \\
& -B\left(\frac{2}{1-\alpha}\right)^{\frac{2 \alpha}{1+\alpha}}\left[(W X)^{\frac{1+\alpha}{4 \alpha}} \pm \sqrt{(W X)^{\frac{1+\alpha}{2 \alpha}}+\frac{4 B}{1-\alpha}}\right]^{-\frac{2 \alpha}{1+\alpha}} .
\end{aligned}
$$

The corresponding EoS parameter is

$$
\omega=-\frac{\alpha}{\alpha-1}-4 B\left(\frac{1}{1-\alpha}\right)^{2}\left[(W X)^{\frac{1+\alpha}{4 \alpha}} \pm \sqrt{(W X)^{\frac{1+\alpha}{2 \alpha}}+\frac{4 B}{1-\alpha}}\right]^{-2}
$$

Using Eq.(2.15) and after some manipulation, the EoS parameter takes the form

$$
\omega_{+}=-\frac{\alpha}{\alpha-1}-\frac{B\left(2 W \beta^{2} t^{2(\beta-1)}\right)^{\frac{1+\alpha}{2 \alpha}}}{\left[(\alpha-1)\left(W \beta^{2} t^{2(\beta-1)}\right)^{\frac{1+\alpha}{2 \alpha}}-2^{\frac{1+\alpha}{2 \alpha}} B\right]^{2}} .
$$

Figure 5 shows the same behavior of EoS parameter as discussed for $n=2$. However, this remains in the region, $-1<\omega_{+}<-1 / 3$, at different points. In this case, the EoS parameter becomes polytropic k-essence EoS parameter as $\alpha<0$. It is concluded that this kind of parameter shows the accelerating expansion of the universe.

\subsubsection{Example 5: $n=-1$}

For this example, $A$ and $\alpha$ satisfy the relation

$$
A=-\frac{2 \alpha}{2 \alpha+1} \quad \text { or } \quad \alpha=-\frac{A}{2(1+A)} .
$$

Consequently, Eq.(3.5) becomes

$$
(1+A) \rho^{1+\alpha}-(W X)^{-\frac{1+\alpha}{2 \alpha}} \rho^{-(1+\alpha)}-B=0,
$$




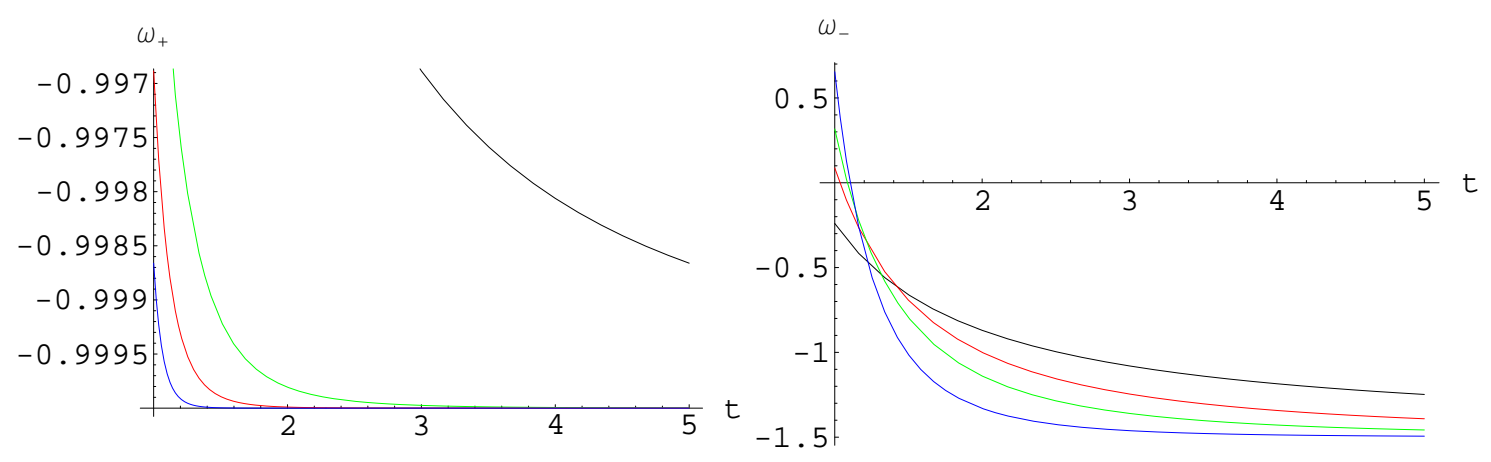

Figure 6: Plot of $\omega$ versus $t$ with $W=2$ and $B=-1$. In the left graph, $\beta>0, \alpha=$ 1.5 and in right graph $\beta<0, \alpha=-1.5$.

which yields the solution

$$
\rho=\left[\frac{B \pm \sqrt{B^{2}+4(1+A)(W X)^{-\frac{1+\alpha}{2 \alpha}}}}{2(1+A)}\right]^{\frac{1}{1+\alpha}} .
$$

or

$$
\rho=\left[\frac{B(2 \alpha+1)}{2} \pm \frac{2 \alpha+1}{2} \sqrt{B^{2}+\frac{4}{2 \alpha+1}(W X)^{-\frac{1+\alpha}{2 \alpha}}}\right]^{\frac{1}{1+\alpha}} .
$$

The pressure is

$$
\begin{aligned}
& p=-\frac{2 \alpha}{2 \alpha+1}\left[\frac{B(2 \alpha+1)}{2} \pm \frac{2 \alpha+1}{2} \sqrt{B^{2}+\frac{4}{2 \alpha+1}(W X)^{-\frac{1+\alpha}{2 \alpha}}}\right]^{\frac{1}{1+\alpha}} \\
& -B\left[\frac{B(2 \alpha+1)}{2} \pm \frac{2 \alpha+1}{2} \sqrt{B^{2}+\frac{4}{2 \alpha+1}(W X)^{-\frac{1+\alpha}{2 \alpha}}}\right]^{-\frac{\alpha}{1+\alpha}} .
\end{aligned}
$$

The corresponding EoS parameter yields

$$
\omega=-\frac{2 \alpha}{2 \alpha+1}-B\left[\frac{B(2 \alpha+1)}{2} \pm \frac{2 \alpha+1}{2} \sqrt{B^{2}+\frac{4}{2 \alpha+1}(W X)^{-\frac{1+\alpha}{2 \alpha}}}\right]^{-1} .
$$

Inserting the value of kinetic term $X$, the positive and negative roots of EoS parameter $\omega$ become

$$
\begin{aligned}
& \omega_{+}=-\frac{2 \alpha}{2 \alpha+1}-\frac{B^{2}\left(W \beta^{2} t^{2(\beta-1)}\right)^{\frac{1+\alpha}{2 \alpha}}}{B^{2}(2 \alpha+1)\left(W \beta^{2} t^{2(\beta-1)}\right)^{\frac{1+\alpha}{2 \alpha}}+2^{\frac{1+\alpha}{2 \alpha}}}, \\
& \omega_{-}=-\frac{2 \alpha}{2 \alpha+1}+B^{2}\left(\frac{W \beta^{2} t^{2(\beta-1)}}{2}\right)^{\frac{1+\alpha}{2 \alpha}} .
\end{aligned}
$$

The left graph in Figure 6 shows the cosmological evolution of EoS parameter $\omega_{+}$with respect to time. It represents a quintessence region of the universe for 
higher values of $\beta$ while for smaller values, it becomes constant and behaves like cosmological constant. In the right graph, polytropic k-essence EoS parameter is plotted. It represents initially a matter dominated universe for $t<1.2$ for smaller values of $\beta$. Then $\omega_{-}$crosses the phantom divide line from quintessence to phantom region and becomes constant in the phantom region $\left(\omega_{-}<-1\right)$.

\section{Other Forms of k-essence Cosmology}

There are two other possibilities of solvable k-essence cosmology [12] such as:

- General k-essence cosmology: $K(X, \phi)=V(\phi) F(X)$

- Quintessence k-essence cosmology: $K(X, \phi)=V(\phi)+F(X)$

These forms yield the same equations (3.5) and (3.6) for $\rho$ and for parameter $n$. It is mentioned here that the above forms show the same behavior as in the case of purely kinetic k-essence cosmology.

Finally, we would like to mention the other class of solvable k-essence models namely integrable k-essence models [20]. As an example, let us consider the following so-called $\mathrm{E}_{I I E}$-model

$$
K_{X X}=2 K^{3}+X K+\alpha(\phi),
$$

where $\alpha=\alpha(\phi)$. It is nothing but the Painlevé II (P-II) equation which is known as integrable. Let the solution of this equation we define as $K=K(X ; \alpha)$ and here we assume that $\alpha=$ constant. Then the equation (4.1) has the following particular solutions (see [20] and references therein)

$$
\begin{aligned}
K & \equiv K(X ; 1.5)=\psi-\left(2 \psi^{2}+X\right)^{-1}, \\
K & \equiv K(X ; 1)=-\frac{1}{X}, \\
K & \equiv K(X ; 2)=\frac{1}{X}-\frac{3 X^{2}}{X^{3}+4}, \\
K & \equiv K(X ; 3)=\frac{3 X^{2}}{X^{3}+4}-\frac{6 X^{2}\left(X^{3}+10\right)}{X^{6}+20 X^{3}-80}, \\
K & \equiv K(X ; 4)=-\frac{1}{X}+\frac{6 X^{2}\left(X^{3}+10\right)}{X^{6}+20 X^{3}-80}-\frac{9 X^{5}\left(X^{3}+40\right)}{X^{9}+60 X^{6}+11200}, \\
K & \equiv K(X ; 0.5 \epsilon)=-\epsilon \psi
\end{aligned}
$$

and so on. Here

$$
\psi=(\ln \chi)_{X}, \quad \chi(X, \phi)=C_{1}(\phi) A i\left(-2^{-1 / 3} X\right)+C_{2}(\phi) B i\left(-2^{-1 / 3} X\right),
$$

where $C_{i}=C_{i}(\phi)$ and $A i(x), B i(x)$ are Airy functions. Each of these solutions determine different type of cosmology. For example, the solution (4.4) corresponds to the EoS:

$$
\begin{aligned}
p & =\frac{2\left(2-X^{3}\right)}{X\left(X^{3}+4\right)} \\
\rho & =\frac{6\left(X^{6}-10 X^{3}-8\right)}{X\left(X^{3}+4\right)^{2}} .
\end{aligned}
$$


Hence we get the EoS parameter

$$
\omega=-\frac{1}{3}-\frac{4 X^{3}}{X^{6}-10 X^{3}-8} .
$$

Inserting the value of kinetic term (2.15), this equation takes the form

$$
\omega=-\frac{1}{3}-\frac{32 \phi_{0}^{6} \beta^{6} t^{6(\beta-1)}}{\phi_{0}^{12} \beta^{12} t^{12(\beta-1)}-80 \phi_{0}^{6} \beta^{6} t^{6(\beta-1)}-512} .
$$

If $\beta=1=\phi_{0}$, then $\omega \sim-0.27$. This shows that the solution (4.4) represents a universe which is nearly to meet the quintessence phase of the accelerating universe. Similarly, we can calculate the EoS and its parameter for the other solutions from the system (4.2)-(4.7) and as well as for the other integrable k-essence models from [20]. Finally we note that it is interesting to extend results of this work to the periodical and quasi-periodical gas/fluid models (see e.g. Ref. [21]) as well as to the knot universe models [22].

\section{Conclusion}

It has always been interesting to explore models to study the accelerating expansion of the universe. This paper is devoted to the study of some solvable k-essence cosmologies using MCG. In particular, we have discussed the purely kinetic k-essence model by taking the energy density and pressure of MCG model. We have found a nonlinear differential equation for the energy density which depends upon the parameter $n$. We have constructed EoS parameter for different values of $n$ by using power law form of potential. Also, the values of constants involved in the model such as, $A, B, \alpha$ and $\beta$ are constrained in such a way that they give the compatible behavior of our expanding universe with acceleration. The graphical results can be summarized as follows:

- Example 1 is taken as $n=0$ which further leads to two cases. The case $\alpha=0$ gives accelerating expansion of the universe dominated by cosmological constant for $\beta>0$, while $\beta<0$ indicates a universe when $\mathrm{DE}$ is dominated over the matter to accelerate the universe. The second case, $A=-1$, indicaress the dynamics of the universe underlying in quintessence phase.

- The second example leads to the generalized Chaplygin gas model for $n=1$. For this value of parameter, initially our universe shows quintessence behavior of DE dominated era. After that, it converges to $\omega=-1$ representing cosmological constant.

- For $n=2$ and $\beta<0$, the EoS parameter shows quintessence behavior of the universe.

- The case $n=0.5$, using k-essence polytropic EoS parameter, gives the same dynamics of the universe as discussed for the parameter $n=2$. 
- For $n=-1, \omega_{+}$leads to a cosmological constant dominated universe for smaller values of $\beta$ whereas the k-essence polytropic EoS parameter $\omega_{-}$represents a quintom behavior and becomes constant in the phantom region.

It is concluded that this relationship provides consistent results about accelerating expansion of the universe [13]. We would like to mention here that the same behavior is observed for the general k-essence and quintessence cosmologies as discussed for puerly kinetic k-essence cosmology by using MCG model. Finally, we have given some integrable k-essence cosmologies $K(X, \alpha)$. These models show different phases of the accelerating universe depending upon its parameters.

\section{References}

[1] Perlmutter, S. et al.: Astrophys. J. 517(1999)565.

[2] Carroll, S.M.: Phys .Rev. Lett. 81(1998)3067.

[3] Yadav, A.K.: Astrophys. Space Sci. 335(2011)565.

[4] Khatua, P.B., Chakraborty, S. and Debnath, U.: arXiv:1105.3393.

[5] Bento, M.C., Bertolami, O. and Sen, A.A.: Phys. Rev. D66(2002)043507.

[6] Matarrese, S. et al.: Dark Matter and Dark Energy (Springer, 2010).

[7] Mukhanov, V., Armendariz-Picon, C. and Steinhardt, P.J.: Phys. Rev. Lett. $\mathbf{8 5}(2010) 4438$.

[8] Piazza, F. and Tsujikawa, S.: JCAP 07(2004)004.

[9] Yerzhanov, K.K. et al.: arXiv:1012.3051.

[10] Razina, O. et al.: Eur. Phys. J. Plus 85(2011)126.

[11] Tsyba, P. et al.: arXiv:1103.5918.

[12] Myrzakulov, R.: arXiv:1011.4337.

[13] Jamil, M. et al.: Astrophys. Space Sci. 336(2011)325.

[14] Garriga, J. and Mukhanov, V.F.: Phys. Lett. B458(1999)219.

[15] Scherrer, R.: Phys. Rev. Lett. 93(2004)011301.

[16] De Putter, R. and Linder E.V.: Astropart. Phys. 28(2007) 263.

[17] Benaoum H.B.: arXiv:0205140v1.

[18] Jamil, M., Hussain, I. and Momeni, D.: Eur. Phys. J. Plus 126(2011)80.

[19] Jamil, M., Rahaman, F. and Kalam, M.: Eur. Phys. J. C60(2009)149. 
[20] Myrzakul Sh.R., Esmakhanova K.R., Myrzakulov K.R., Nugmanova G.N., Myrzakulov R.: arXiv:1105.2771.

[21] Bamba K., Yesmakhanova K., Yerzhanov K., Myrzakulov R.: arXiv:1203.3401; Bamba K., Debnath U., Yesmakhanova K., Tsyba P., Nugmanova G., Myrzakulov R.: arXiv:1203.4226;

Bamba K., Razina O., Yerzhanov K., Myrzakulov R.: arXiv:1203.2804.

[22] Myrzakulov R.: arXiv:1008.4486;

Yesmakhanova K., Myrzakulov Y., Nugmanova G., Myrzakulov R.: International Journal of Theoretical Physics, 51 (2012) 1204-1210, arXiv:1102.4456,

Myrzakulov R.arXiv:1205.5266;

Myrzakulov R.arXiv:1207.1039;

Myrzakulov R. et al.: arXiv:1201.4360;

Myrzakulov R.: arXiv:1204.1093. 